\title{
Automatic detection of the macula in retinal fundus images using multilevel thresholding
}

\author{
Jiri Minar, Kamil Riha, Ales Krupka, Hejun Tong
}

\begin{abstract}
The paper proposes a novel method for detection of macula in medical image of human eye - retinal fundus images that can be used in ophthalmology for detecting various eye's diseases such glaucoma, diabetic retinopathy or macula oedema. The method utilizes an approach of multilevel thresholding of red channel of fundus retinal image. Subsequently, the thresholded layers are preprocessed by application of median blur filter. Algorithm for ellipse detection from OpenCV library is used on all thresholding level. Then proposed technique analyzes detection and evaluates position of macula in fundus image. The precision of the method is evaluated on dataset from public fundus image library DRIVE. The results were 90\% (36 of 40). False detections ware only in images where macula was not visible.
\end{abstract}

Keywords- Automatic, Fundus, Macula, Medical Image processing, Multilevel Segmentation, Thresholding.

\section{INTRODUCTION}

$\mathbf{T}$ HE human eye is one of most important part of human body and diseases like glaucoma, diabetic retinopathy and macular degeneration can irreversible damage human vision. Diabetic retinopathy is usually main reason for the blindness in elder age people if not treated in early stages [1]. Patient's retinal image has to be analyzed for detection of disease like a diabetic retinopathy.

Retinal images are usually known as Fundus images. These images have usually red tint due to rich blood supply. Fundus image contains optical disk, macula and blood vascular, which are important for diagnosis by ophthalmologist [2]. Macula is an oval shaped highly pigmented yellow spot usually located in the center of retina of the human eye. Near its center there is the fovea, a small pit that contains the largest concentration of cone cells in the eye and is responsible for central, high resolution vision. Macula is the darkest region in neighborhood of optical disk in Fundus image Fig. 1. There are also visible vessels and veins. Extraction of retinal

Jiri Minar is with Dept. of Telecommunications, Faculty of Electrical Engineering and Communication, Brno University of Technology, Technicka 12, 61600 Brno, Czech Republic, email: jiri.minar@phd.feec.vutbr.cz

Kamil Riha received his M.Sc. degree in Electronics \& Communication in 2003 and the Ph.D. degree in 3D Scene Acquisition for Auto-Stereoscopic Display in 2007. Presently, he is employed at Brno University of Technology, Faculty of Electrical Engineering, Department of Telecommunications, as the academic employee (since 2006). His research interests include in particular areas of digital image processing and electronics.email: rihak@feec.vutbr.cz

Ales Krupka is with Dept. of Telecommunications, Faculty of Electrical Engineering and Communication, Brno University of Technology, Technicka 12, 61600 Brno, Czech Republic, vascular can be also used in some application related to secure area like personal identification. The main concern of this paper is to detect macula in Fundus imaged. Detection of macula is the most important step for the treatment of macula oedema. Patients with macula oedama have blood leakage in blood vessels in macula area, if detected it can be treated with laser [3].

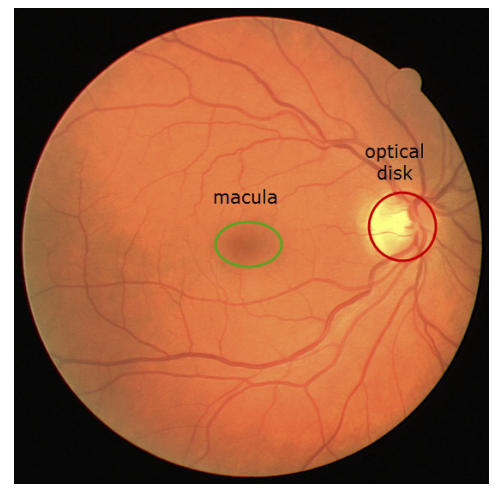

Fig. 1. Retinal image with marked macula and optic disk

\section{RECENT SOLUTION}

Different approaches are presented in literature for macula detection. In [4], macula is detected using line operator. Paper [5] is presenting a method for macula detection using seeded mode tracking approach. Paper [6] presents the algorithm to detect macula using edge detection and region growing techniques. Ant Colony Optimization based hybrid method is presented in [7]. In paper [8] macula is localized first by making use of localized optic disc center and enhanced blood vessels. Finally, macula is detected by taking the distance from center of optic disk and thresholding then combining it with enhanced blood vessels image to locate the darkest pixel in this region, making clusters of these. The article [9] represents an approach of image segmentation on microscopic bone marrow images by using multilevel tresholding technique. This technique has been applied into two types of images which is normal bone marrow and Acute Lymphoblastic Leukemia (ALL) or this paper [10] introduces detection method based on multilevel-thresholding of input image and subsequent analysis of objects shape in each level. Detection algorithm focuses on path tracking of persons in video sequences correct evaluation of tailgating or piggybacking. This paper [11] introduces solution for localization of optic disc by using specialized template matching and segmentation by a deformable contour model. Difference approach of detection in medical images is 
described in this article [12], where is described method for highly accurate and effective localization of the transverse section of the carotis communis artery in ultrasound images using Viola-Jones detector.

The presented solution focuses on macula detection using multi-level thresholding without any supporting detection of area in fundus image. Proposed algorithm should be very accurate with detection of macula center with non-complex algorithm.

\section{Proposed TeCHNIQUE}

The proposed macula detection algorithm using multilevel thresholding and ellipse detection from all level is described in flow chart in Fig. 2. The technique consists of three main parts: Layer selection and image preprocessing, multilevel thresholding and position detection algorithm.

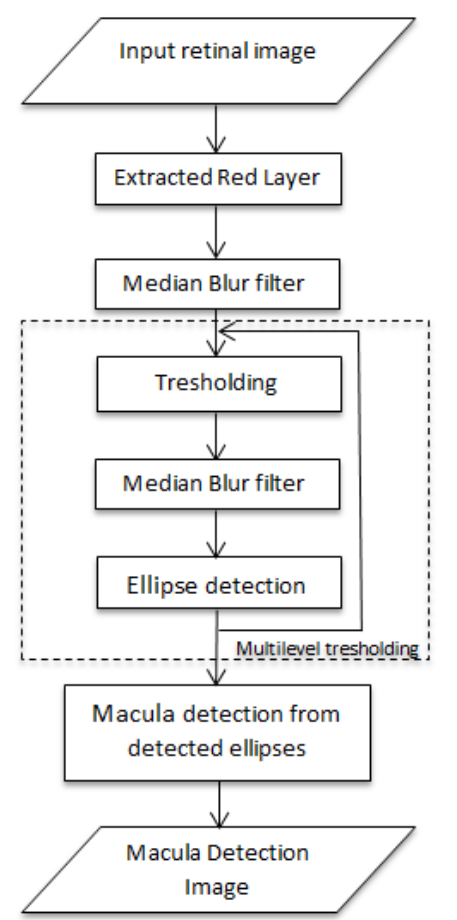

Fig. 2. Complete algorithm for macula detection

\section{A. Layer selection}

The proposed technique uses multilevel thresholding of Fundus image and ellipse detection using OpenCV ${ }^{1}$ (Open Computer Vision) framework. OpenCV contains various libraries for image processing and object detection.

All color channels from Fundus image (red, green, blue) (as seen in Fig. 3) were extracted. Green channel is usually used for vessels and veins segmentation, because there is most image information with veins and vessels. We used red channel as an input point of our algorithm. This decision was made after an analysis of RGB channels. Red channel contains mostly information about macula and less information about veins and vessels (Fig. 3c), which can negatively influence algorithm for ellipse detection.

\footnotetext{
${ }^{1}$ Available from URL: http: / / opencv. org
}

\section{B. Preprocessing}

Macula does not have usually perfect rounded circle or ellipse shape, but it has ellipse with craggy perimeter. For this reason is red channel of fundus image preprocessed by application of blur function using normalized box filter. Thus preprocessed image is used as input to multilevel segmentation and ellipse detection algorithm.
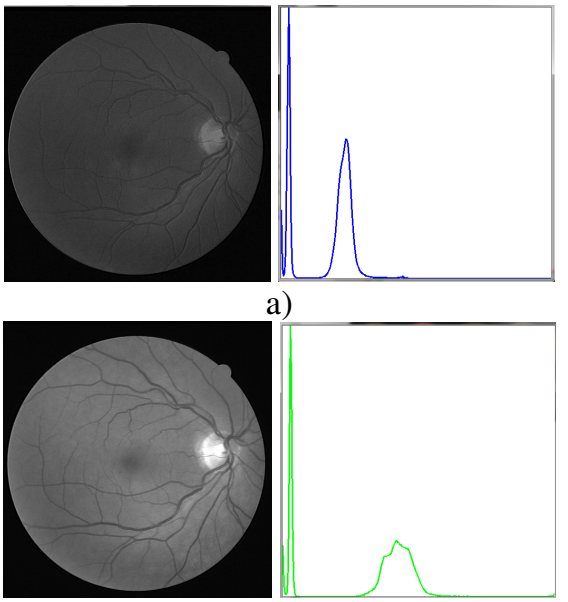

b)

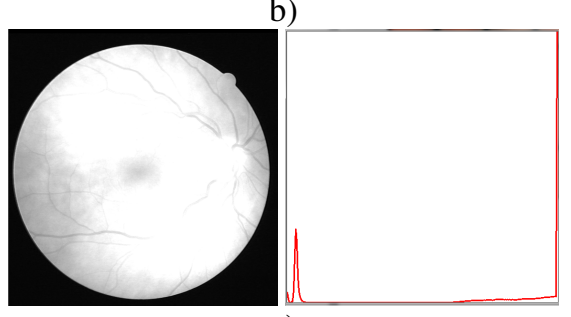

c)

Fig. 3. RGB channels of fundus retinal image with histograms a) Blue b) Green c) Red

\section{Multilevel thresholding}

Consequently the fundus image is segmented in the loop, where loop is created over all threshold level. This multilevel thresholding is computed for all 256 levels, because red channel is processed as grey image with 8-bit information about grey shade. Thresholding levels from 100 to 230 usually return the most useful and quality output for next contour and ellipse detection (as shown in Fig.4).

Thresholding layers are blur again to increase roundness of shape on images to increase change of detection of contour and after that detection of ellipses from this contour.

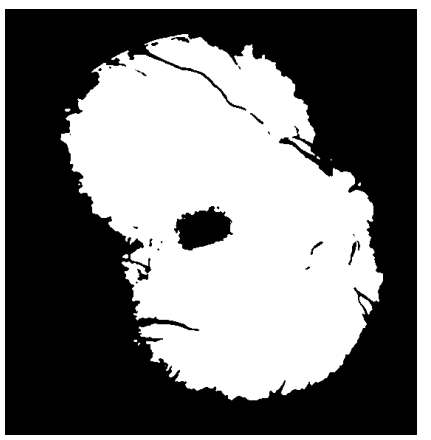

Fig. 4. Thresholding of red channel from fundus image. 


\section{Ellipse detection}

Ellipse contours and shapes is finding in all thresholds level by functions from OpenCV framework. Function for contour detection using algorithm described in this paper [13]. All detected ellipses are evaluated for shape and ellipses eccentricity value lower than 0.5 or greater than 1.5 are eliminated. This elimination is essential for elimination of false detection, because macula is circle shaped or ellipse with small eccentricity as shown in Fig. 5. All ellipses detected in each thresholding level are stored in buffer for consequence processing.

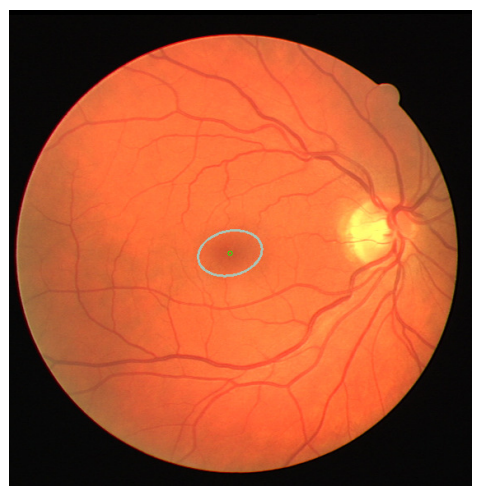

Fig. 5. Detected ellipse from one thresholding level (fig. 4.) and displayed in original fundus image with marked center.

\section{E. Ellipse detection evaluation}

Next step in proposed technique for macula detection is evaluation of all detected ellipses and decision where macula is situated in fundus image. The algorithm 1 shows principle of this detection. First all detected ellipses (Fig. 7) are split into group with the same coordinates of centroid (center of ellipse) with some toleration. We have used toleration of 15 pixels. The group with the biggest amount of detection is marked as group with possible macula location. The biggest ellipse in this group is marked as final detection and shape and centroid is drawn into original image (Fig. 5).

\author{
foreach threshold level do \\ I apply blur filter; \\ I detect contours; \\ I detect ellipse; \\ I eliminate ellipses with wrong eccentricity; \\ I store in buffer; \\ end \\ for $k=0$ to numbers of all detected ellipses do \\ I collect ellipse to group with similar centroid; \\ end \\ evaluate group with most ellipse detection; \\ get coordinates of the biggest ellipse in group; \\ get perimeter of the biggest ellipse in group;
}

Algorithm 1: Evaluation of macula location

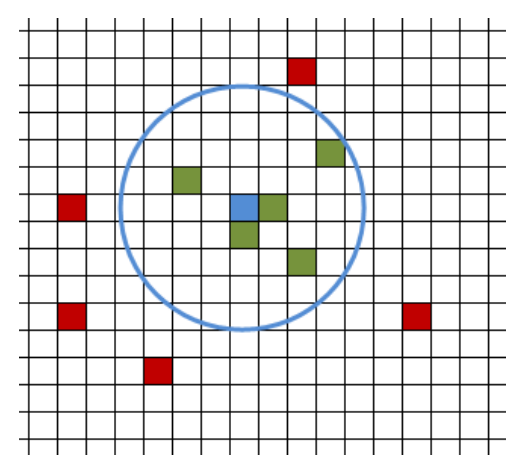

Fig. 7. Grouping of detected ellipses by centroid - with radius evaluation \pm 4 pixels. Blue pixel - initial centroid, green pixels - coordinates of centroids in radius, red pixels - centroids outside radius

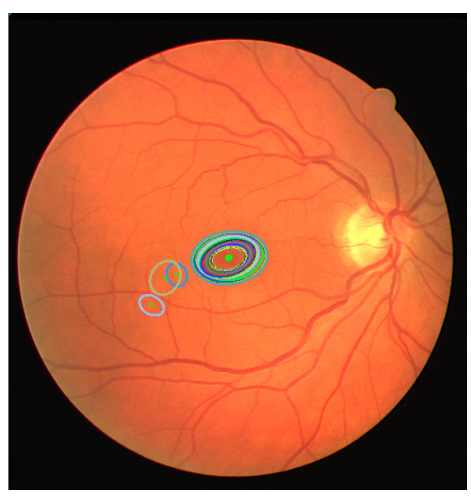

Fig. 8. All ellipses detected for all thresholding levels and displayed in original fundus image with marked center.

\section{EXPERIMENTAL RESULTS}

To evaluate the performance of proposed algorithm, a dataset of 40 images are used. Evaluation is applied on DRIVE (Digital Retinal Images for Vessel Extraction) that is publically available. 40 images of size $565 \times 584$, each pixel having eight bits are included in DRIVE database [14].

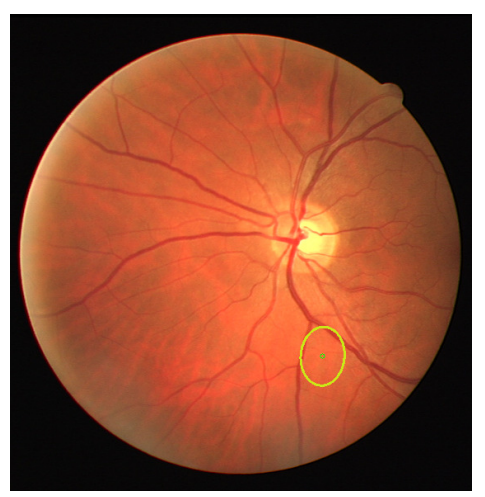

Fig. 9. One of four picture removed from dataset due macula is not presented in this image.

Four images were manually eliminated from this DRIVE dataset of 40 images, because macula is not visible on these images (can even not be seen by human eyes). This can be cause by some eye disease in advanced stage or wrongly focused of fundus image with optical disc in the middle as can be seen in Fig. 8. In this picture there is also marked position, 
where macula is detected - it is false detection - because macula is not presented in image. The algorithm has to designate best detection in the image.

Detection results are very promising. Overall results for whole DRIVE database are $90 \%$ of accurate detection macula in fundus image. Macula was detected in 36 fundus images out of 40. Macula was marked on random places in 4 images. The reason of this false detection macula was caused that macula was not visible at all on these 4 images also by human eye, thus algorithm marked place with the most false detection. This is the place for improvement of algorithm.

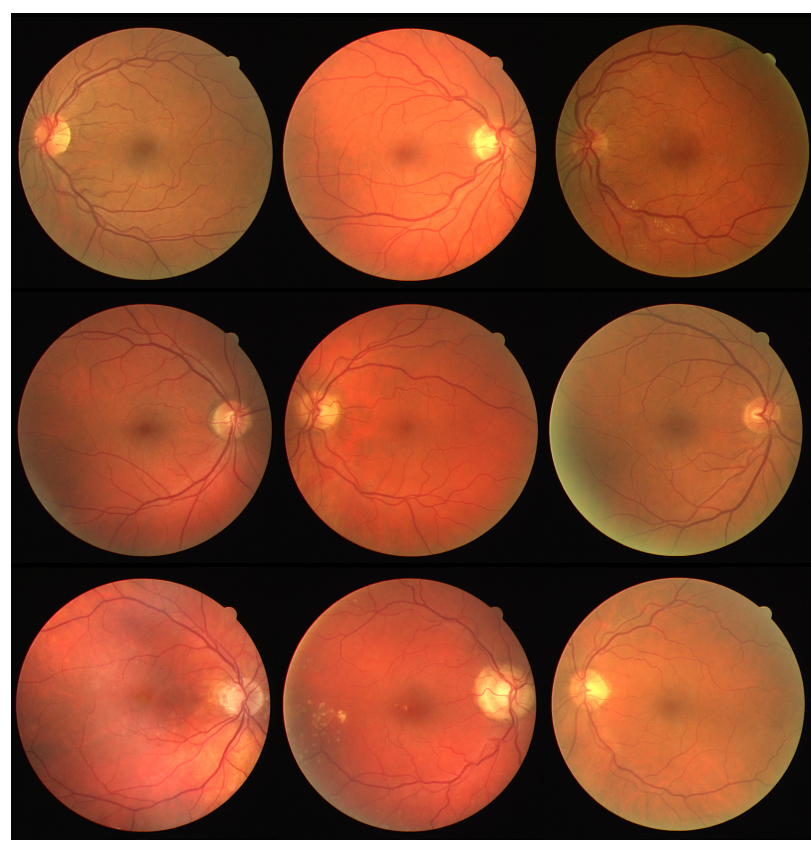

a)

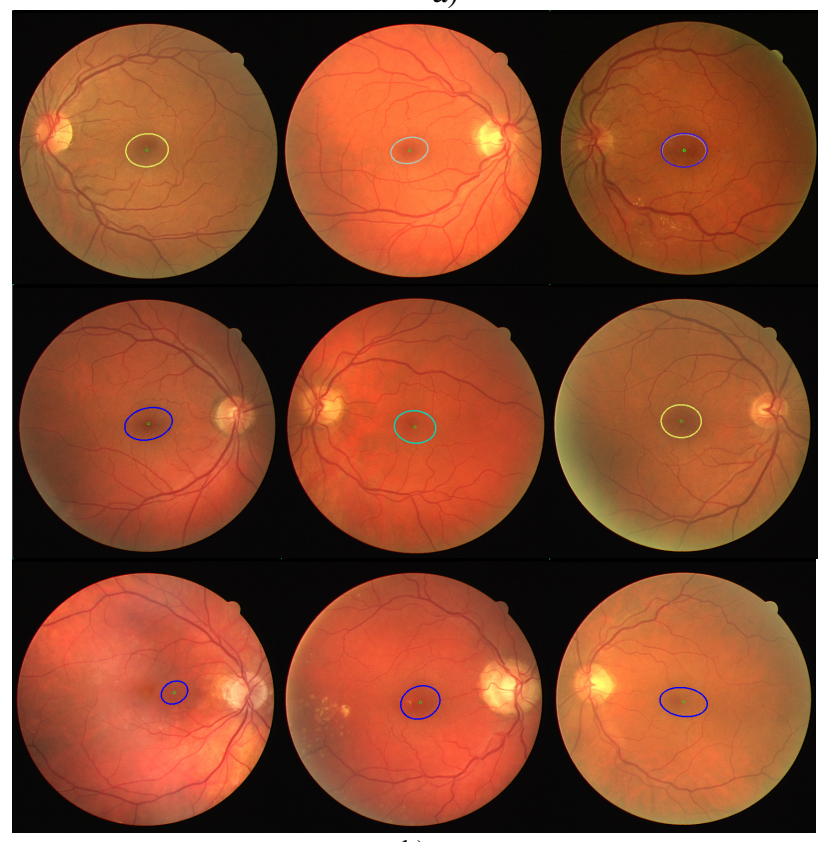

Fig. 10. Nine examples of fundus images from DRIVE database a) Original Fundus images

b) Fundus images with marked detection of macula

\section{CONCLUSION}

We have presented technique for detection of macula position in fundus images. This technique is using multilevel thresholding, contour, and ellipse detection. Proposed method was very successful on test dataset from DRIVE database (40 fundus images). Macula was successfully located on all images where was presented and we detected macula area with $90 \%$ effectivity (36 of 40). False detection were only in images where macula was not visible, thus area where most ellipses were detected, was marked as macula. However, this false detection can be avoided by suitable technique of fundus image collection or enhance of the algorithm by not displaying "weak" detection e.g. area with very small ellipse detection.

\section{REFERENCES}

[1] G.E. Lang, "Diabetic retinopathy", Development in Ophthalmology, Vol. 39, 2007.

[2] K. Akita, H. Kuga, "A computer method of understanding ocular fundus image," Pattern Recognition, vol. 15, no. 6, pp 431-443, 1982.

[3] Chinenye, "Discover the amazing way to treat and handle sickness \& diseases", Vol.2, 2007

[4] Shijian Lu, Joo Hwee Lim, Automatic macula detection from retinal images by a line operator", Proceedings of 2010 IEEE 17th International Conference on Image Processing, pp. 4073-4076, 2010.

[5] Damon W.K. Wong, Jiang Liu, Ngan-Meng Tan, Fengshou Yin, Xiangang Cheng, Ching-You Cheng, Gemmy C.M. Cheung, Tien Yin Wong, "Automatic Detection of the Macula in Retinal Fundus Images using Seeded Mode Tracking Approach", 34th Annual International Conference of the IEEE EMBS, pp. 4950-4953, 2012

[6] C.Marin, S. PenaA, M.G.Penedo, M. Ortega, J. Rouco, A. P. Reino, M. Pena, "Macula Precise Localization Using Digital Retinal Angiographies", WSEAS Transactions on Computer Research, Issue 1 vol 3., pp.21-33, January 2008.

[7] Kavitha, G. Ramakrishnan, "Identification and analysis of macula in retinal images using Ant Colony Optimization based hybrid method", World Congress on Nature \& Biologically Inspired Computing, pp 1174 - 1177, Dec 2009.

[8] Maryam Mubbashar, Anam Usman, M. Usman Akram, Automated System for Macula Detection in Digital Retinal Images", Information and Communication Technologies (ICICT), 2011 International Conference, pp. 1-5, 2011

[9] R. Adollah, E. U. Francis, M. Y. Mashor, N. H. Harun, "Bone Marrow Image Segmentation Based on Multilevel Thresholding", 2012 International Conference on Biomedical Engineering (ICoBE), pp. $458-61,2012$

[10] Minar J., Riha K., Tong H., "Intruder detection for automated access control system with Kinect device", 36th International Conference on Telecommunications and Signal Processing (TSP), pp. 826-829, 2012

[11] Lowell, J., Hunter, A., Steel, D., Ryder, B., Fletcher, E. "Optic Nerve Head Segmentation" IEEE Transactions on Medical Imaging, 23 (2), Feb 2004.

[12] Riha K., Masek J., Burget R., Benes R., Zavodna E., "Novel Method for Localization of Common Carotid Artery Transverse Section in Ultrasound Images Using Modified Viola- Jones Detector", Ultrasound in medicine and biology, vol. 39, pp. 1887-1902, 2013

[13] Suzuki, S. and Abe, K., "Topological Structural Analysis of Digitized Binary Images by Border Following", CVGIP 30 1, pp 32-46 (1985)

[14] J.J. Staal, M.D. Ambramoff, M. Niemeijer, M.A. Viergever, B. van Ginneken, "Ridge based vessel segmentation in color images of the retina", IEEE Transactions on Medical Imaging, vol. 23, pp. 501-509, 2004 